\title{
Machine Learning Algorithms to Distinguish Myocardial Perfusion SPECT Polar Maps
}

\begin{abstract}
Erito Marques de Souza Filho ${ }^{1,2 *}$, Fernando de Amorim Fernandes ${ }^{1,3}$, Christiane Wiefels ${ }^{1,4}$, Lucas Nunes Dalbonio de Carvalho ${ }^{2}$, Tadeu Francisco dos Santos ${ }^{1}$, Alair Augusto Sarmet M. D. dos Santos ${ }^{1}$, Evandro Tinoco Mesquita ${ }^{1}$, Flávio Luiz Seixas ${ }^{5}$, Benjamin J. W. Chow ${ }^{4}$, Claudio Tinoco Mesquita ${ }^{1,6}$ and Ronaldo Altenburg Gismondi ${ }^{1}$

${ }^{1}$ Post-graduation in Cardiovascular Sciences, Universidade Federal Fluminense, Niterói, Rio de Janeiro, Brazil, ${ }^{2}$ Department of Languages and Technologies, Universidade Federal Rural do Rio de Janeiro, Rio de Janeiro, Brazil, ${ }^{3}$ Department of Nuclear Medicine, Hospital Universitário Antônio Pedro/EBSERH, Universidade Federal Fluminense, Rio de Janeiro, Brazil, ${ }^{4}$ Department of Cardiac Image, University of Ottawa Heart Institute, Ottawa, ON, Canada, ${ }^{5}$ Institute of Computing, Universidade Federal Fluminense, Rio de Janeiro, Brazil, ${ }^{6}$ Department of Nuclear Medicine, Hospital Pró-Cardíaco, Americas Serviços Medicos, Rio de Janeiro, Brazil
\end{abstract}

Myocardial perfusion imaging (MPI) plays an important role in patients with suspected

OPEN ACCESS

Edited by:

Marcus R. Makowski, Technical University of

Munich, Germany

Reviewed by:

Antti Saraste,

University of Turku, Finland

Ernst Wellnhofer,

Charité - Universitätsmedizin

Berlin, Germany

*Correspondence: Erito Marques de Souza Filho mederitomarques@gmail.com

Specialty section:

This article was submitted to

Cardiovascular Imaging,

a section of the journal

Frontiers in Cardiovascular Medicine

Received: 15 July 2021 Accepted: 29 September 2021 Published: 11 November 2021

Citation:

de Souza Filho EM, Fernandes FdA, Wiefels C, de Carvalho LND, dos

Santos TF, dos Santos AASMD,

Mesquita ET, Seixas FL, Chow BJW, Mesquita CT and Gismondi RA (2021)

Machine Learning Algorithms to Distinguish Myocardial Perfusion

SPECT Polar Maps.

Front. Cardiovasc. Med. 8:741667.

doi: 10.3389/fcrm.2021.741667 and documented coronary artery disease (CAD). Machine Learning (ML) algorithms have been developed for many medical applications with excellent performance. This study used ML algorithms to discern normal and abnormal gated Single Photon Emission Computed Tomography (SPECT) images. We analyzed one thousand and seven polar maps from a database of patients referred to a university hospital for clinically indicated MPI between January 2016 and December 2018. These studies were reported and evaluated by two different expert readers. The image features were extracted from a specific type of polar map segmentation based on horizontal and vertical slices. A senior expert reading was the comparator (gold standard). We used cross-validation to divide the dataset into training and testing subsets, using data augmentation in the training set, and evaluated $04 \mathrm{ML}$ models. All models had accuracy $>90 \%$ and area under the receiver operating characteristics curve (AUC) $>0.80$ except for Adaptive Boosting ( $A \cup C=0.77$ ), while all precision and sensitivity obtained were $>96$ and 92\%, respectively. Random Forest had the best performance (AUC: 0.853; accuracy: 0,938; precision: 0.968; sensitivity: 0.963). ML algorithms performed very well in image classification. These models were capable of distinguishing polar maps remarkably into normal and abnormal.

Keywords: machine learning, polar maps, myocardial perfusion imaging (MPI), coronary artery disease, random forest

\section{INTRODUCTION}

Myocardial perfusion imaging (MPI) plays an essential role in the diagnosis and risk stratification of a patient with suspected and documented coronary artery disease (CAD) (1). Thus, accurate reporting of MPI is paramount and requires experienced professionals (2-4). Interpretation errors by health professionals can impact patient care and need to be minimized. The use of MPI is common. For instance, 61.9 studies were performed for every 1,000 Medicare beneficiaries in 2013. In Australia, there were 337 MPI studies per 100,000 people in 11 years (5). High volumes, 
increasing workload, and clinical demands can potentially lead to interpretation errors. Therefore, a decision support tool capable of interpreting could improve efficiency, accuracy, and costs (6).

Artificial intelligence (AI) has the potential to improve healthcare delivery. It combines mathematical models and computation, designed to emulate human intelligence (7). In particular, machine learning (ML), a subset of AI models, encompasses several methods capable of performing tasks after exposure to data (8). It has gained relevance in medicine and has transitioned from structured data to image analysis in diagnostic imaging and MPI (9).

In this context, designing an ML tool to give the physician some support for his MPI reports would be necessary. This tool would be precious for medical residents and other trainees in disagreement between the reader and the algorithm. In case of discordance, in-depth analysis and review will be necessary for the final decision-making, allowing for better comprehension of the method, improved reports' quality, and better training (Figure 1). We evaluated 04 supervised ML algorithms (Adaptive Boosting, Gradient Boosting, Random Forest, and Extreme Gradient Boosting) to distinguish between normal vs. abnormal single-photon emission computed tomography (SPECT) MPI polar maps.

\section{MATERIALS AND METHODS}

We analyzed 1,007 consecutive MPI studies (January 2016December 2018). Studies were acquired in the supine position at stress and rest with additional prone imaging at stress for males to correct for diaphragmatic attenuation artifact. All patients underwent an 8-frame ECG-gated 2 day reststress Tc-99 $\mathrm{m}$ sestamibi myocardial perfusion single-detector SPECT (Millennium MPR, GE Healthcare), according to the

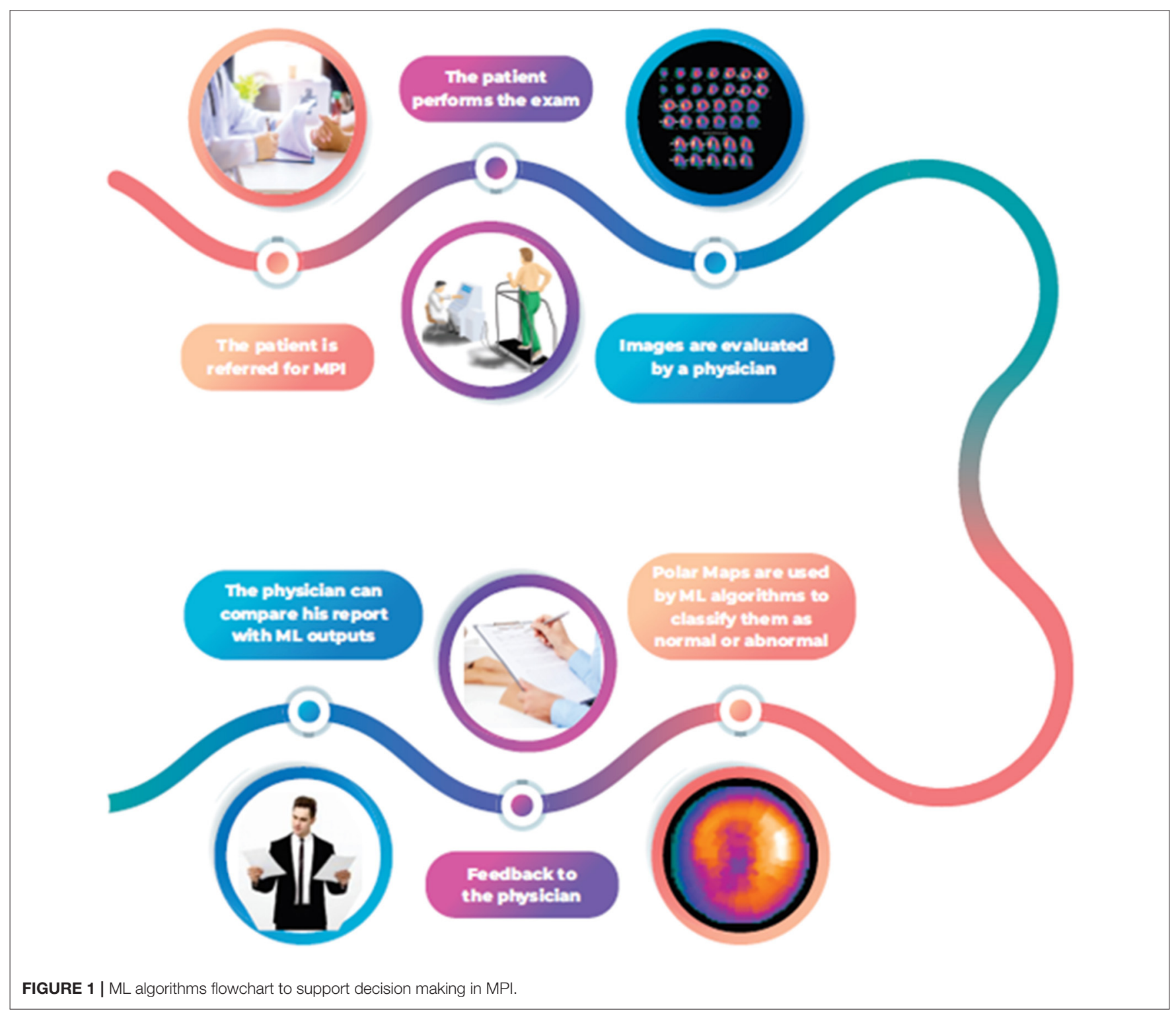


ASNC guidelines (10). Rest-stress doses were determined based on the patient's body weight by a factor of 0.25 $\mathrm{mCi} / \mathrm{kg}$. Acquisition times were $21 \mathrm{~min}$ for stress and rest imaging using a $180^{\circ}$ orbit. We reconstructed the transaxial emission images with ordered-subsets expectation maximization (OSEM) algorithm with 04 subsets and 10 iterations and a uniform initial estimate (11). We used Emory Cardiac ToolboxTM (Emory University/ Syntermed, Atlanta, GA) for image reconstruction, axis orientation, and polar maps. MPI studies were analyzed and reported using all the relevant clinical and stress-derived data. We used Emory Cardiac ToolboxTM for image reconstruction, axis orientation, and polar map generation. Images were classified as normal or abnormal using visual analysis, quantitative parameters, and wall motion data. MPI was considered normal in the presence of normal left ventricular cavity size, normal regional wall motion and left ventricular thickening, homogeneous perfusion throughout the myocardium, a normal left ventricular ejection fraction $(>45 \%)$, and normal right ventricular uptake (12). A single expert reader initially reported these studies, and then a second expert evaluated all the software's polar maps before validating the study. When the studies had conflicting interpretations, the second reader analysis was considered for the ML algorithm. All specialists who participated in the evaluation process have a specialist in nuclear medicine and more than 15 years of experience. The senior researcher responsible for conducting the review and the title of specialist was president of the Brazilian Society of Nuclear Medicine, has over 20 years of experience, and more than 100 articles published in the area. Our work was carried out at Hospital Universitario Antônio Pedro in Brazil, and we only had access to anonymized polar maps in our study. Polar maps were post-processed (GE Healthcare Xeleris $®$ ) and exported in.tiff format with matrix size $175 \times 175$.

So, we generated 02 polar maps (stress/rest) for female patients and 03 (stress/rest/prone) for male patients. We did not use the clinical data for the ML algorithms, but the image attributes such as pixel position and intensity.

In our work, only images from myocardial perfusion were used. The Ethics Committee (Universidade Federal Fluminense) has authorized us to use these images as long

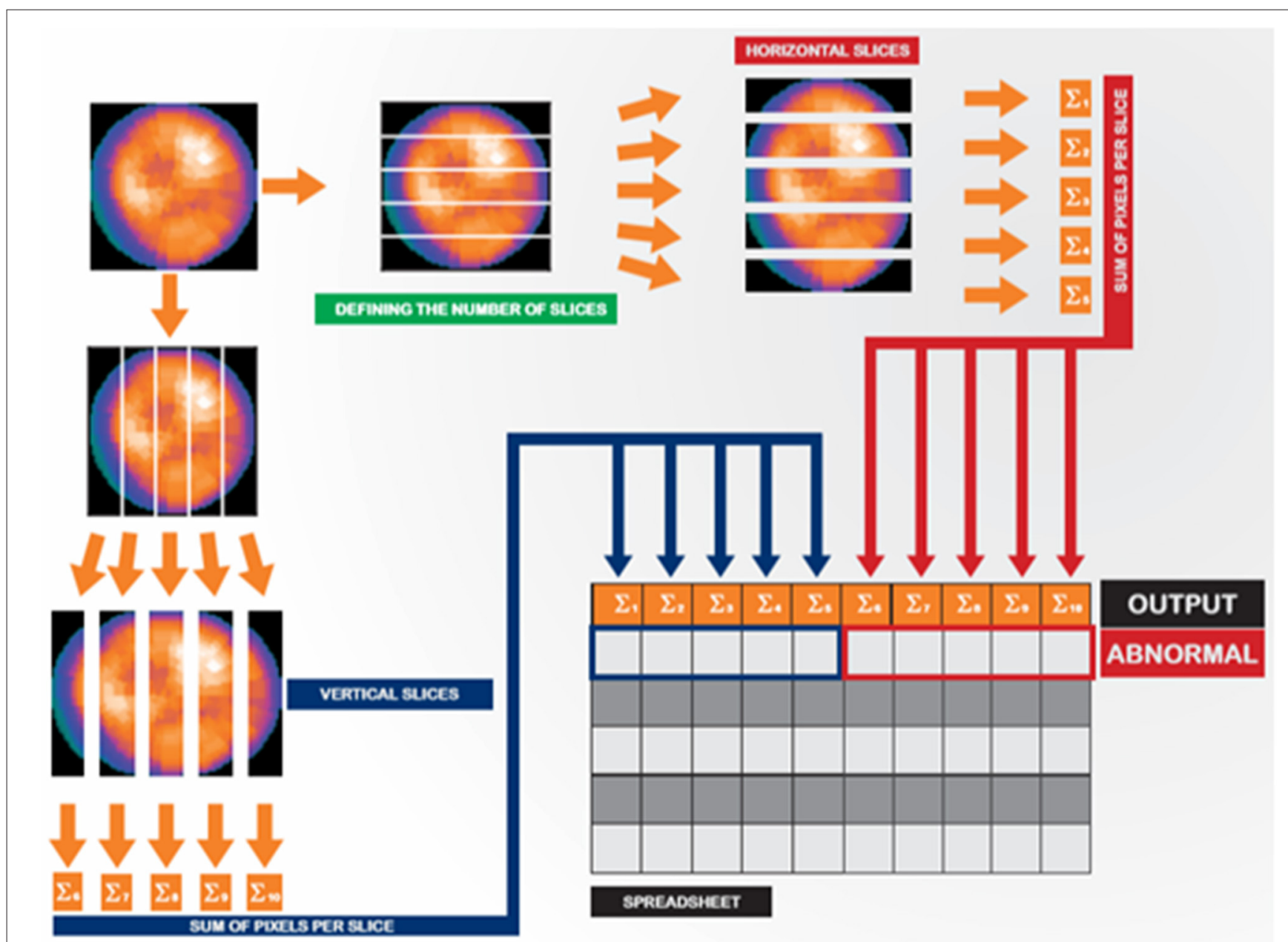

FIGURE 2 | Image slicing and feature extraction strategy. 
as they are anonymized in agreement with the Declaration of Helsinki.

\section{Features Extraction}

We extracted image features using an image slicing process based on Ouali et al. (13). In this process, each image was divided into 05 horizontal and 05 vertical slices (Figure 2), where the pixel intensities from each slice were sum, so we obtained a total of 10 attributes. After processing, cardiac nuclear medicine images are traditionally mapped to a colored single-channel representation (the so-called GE color). Thus, we obtained a matrix with 11 columns per 955 rows (one row for each image). The first 10 columns represent the slicing process features, and the last column corresponds to the label indicating whether the polar map is normal (1) or abnormal (0). We complied with the General Data Protection Regulation (GDPR) (14).

\section{Algorithms}

Four ensemble ML algorithms were used [Adaptive Boosting $(\mathrm{AB})$, Gradient Boosting (GB), Random Forests (RF), and eXtreme Gradient Boosting (XGB)]. We built a set of base classifiers in ensemble models and classified new data by voting for these classifiers' predictions. RF uses bootstrap aggregation (bagging) in the process of constructing the base classifiers. Boosting techniques ( $\mathrm{AB}, \mathrm{GB}$, and $\mathrm{XGB}$ ) were used to properly combine a series of weak classifiers to obtain a stronger one. A weak classifier occurred when a feature's performance was slightly superior to random guessing (15-24). Table A in Section Supplementary Data 1 shows parameters used in our ML algorithms.

We assessed the model's performance using the following metrics: AUC, sensitivity, precision (positive predictive value), and F1 measure (the harmonic mean of the precision and sensitivity). ML algorithms were implemented in Python 3 using open-source libraries $(25,26)$.

\section{Cross-Validation}

We used ten-fold cross-validation to validate the classification model results $(27,28)$. In the cross-validation, the training database is divided into $\mathrm{k}(\mathrm{k}=10)$ parts of the same size, $\mathrm{k}-1$ of which is for training and testing. Therefore, all data were used in the training process. All images used were independent, and there was no data leak in the cross-validation process.

TABLE 1 | Polar maps characteristics.

\begin{tabular}{lcccccc}
\hline & Rest M & Str M & Prone M & Rest F & Str F & Total \\
\hline normal & 10 & 10 & 10 & 39 & 39 & 108 \\
abnormal & 91 & 91 & 91 & 313 & 313 & 899 \\
Total & 101 & 101 & 101 & 352 & 352 & 1,007
\end{tabular}

Rest $M$, rest male; Str $M$, stress male; Prone $M$, prone male; Rest $F$, rest female; $\operatorname{Str} M$, stress female.

\section{Data Augmentation}

The database consisted of 108 normal images and 899 abnormal images, an unbalanced training dataset (Table 1). In this context, we increased the number of normal images using the polar maps' geometric properties. This data augmentation was done only in training dataset splits to avoid leakage of information. 324 new polar maps were generated - three for each normal polar map $\left(3^{*} 108\right)$. Therefore, cross-validation was done with 9 boxes containing 202 images in the test set and a training set with the remaining 1,129 images. In the tenth box, the training set consisted of 196 images. Figure A in Section Supplementary Data 2 shows how the process was done. Data used in the models are available in Supplementary Material.

\section{RESULTS}

In Table 2, we can see the ML algorithms performance. All models had sensitivity $>92 \%$. However, only RF had $96 \%$. AB, GB, and XGB achieved, respectively, 92, 94, 95\%. Sensitivity standard deviation was lower in GB, RF, and XGB (0.02). RF had the best accuracy (93\%), followed by GB (92\%), XGB (92\%), and $\mathrm{AB}(90 \%)$. Precision ranged from 0.96 (XGB, $\mathrm{RF}, \mathrm{AB}$ ) to $0.97(\mathrm{~GB})$ while $\mathrm{F} 1$ measure ranged from $0.94(\mathrm{AB})$ to 0.96 (RF). All precision standard deviations were 0.01 . We achieved the best AUC by RF (0.85), followed by XGB (0.82), GB (0.81), and $A B(0.77)$. AUC standard deviation was lower in GB (0.05). All processing time was lesser than $0.2 \mathrm{~s}$. We obtained the best processing time in $\mathrm{AB}(0.16)$.

\section{DISCUSSION}

This study evaluated ML algorithms' ability to distinguish between normal and abnormal SPECT myocardial perfusion polar maps (without specifying the nature of the abnormality). ML algorithms had high accuracy in image classification. Three models obtained AUC higher than 0.80, and had precision and sensitivity $>0.94$. The performance was also high if we consider the F1 measure. Indeed, these models can contribute significantly to the decision-making process. The results obtained in this work reiterate the role of these algorithms and their importance in nuclear medicine. They add to other previous successful experiences.

An example of this is Nakajima et al., who used artificial neural networks in CAD diagnosis and had impressive results. The AUC was superior to 0.9 (overall) in all cases tested, including patients with previous myocardial infarction and coronary revascularization (29-32). Another example of successful performance (AUC $=0.81$ ) was the use of an ML algorithm (LogitBoost) to predict early revascularization after myocardial perfusion imaging with SPECT $(33,34)$. Cortes $(35)$ had good results $(A U C=0.83$ ) using a different $M L$ algorithm, called Support Vector Machine (SVM), to evaluate a patient's risk of cardiac death after adenosine myocardial perfusion SPECT (1).

It is worth mentioning, as pointed out by Elhendy et al. (36), that a study classified as normal has high relevance regarding a patient's prognosis: the annual mortality and cardiac event 
TABLE 2 | Ensemble ML algorithms performance (mean and standard deviation tenfold cross validation results).

\begin{tabular}{|c|c|c|c|c|c|c|}
\hline Model & Accuracy & $\mathbf{F 1}$ & Precision & Sensitivity & Time (s) & \\
\hline \multicolumn{7}{|c|}{ Training set: Mean +/- Standard Deviation } \\
\hline$A B$ & $0.961+/-0.006$ & $0.970+/-0.004$ & $0.982+/-0.004$ & $0.959+/-0.005$ & $0.175+/-0.023$ & \\
\hline GB & $0.993+/-0.001$ & $0.995+/-0.001$ & $0.999+/-0.001$ & $0.991+/-0.002$ & $0.411+/-0.043$ & \\
\hline $\mathrm{RF}$ & $1.000+/-0.000$ & $1.000+/-0.000$ & $1.000+/-0.000$ & $1.000+/-0.000$ & $0.297+/-0.046$ & \\
\hline XGB & $1.000+/-0.000$ & $1.000+/-0.000$ & $1.000+/-0.000$ & $1.000+/-0.000$ & $0.174+/-0.018$ & \\
\hline Model & Accuracy & $\mathbf{F 1}$ & Precision & Sensitivity & Time (s) & AUC \\
\hline \multicolumn{7}{|c|}{ Test set: Mean +/- Standard Deviation } \\
\hline$A B$ & $0.907+/-0.032$ & $0.947+/-0.020$ & $0.969+/-0.014$ & $0.927+/-0.044$ & $0.164+/-0.044$ & $0.778+/-0.068$ \\
\hline GB & $0.927+/-0.020$ & $0.959+/-0.012$ & $0.970+/-0.012$ & $0.949+/-0.027$ & $0.416+/-0.056$ & $0.815+/-0.059$ \\
\hline $\mathrm{RF}$ & $0.938+/-0.017$ & $0.965+/-0.010$ & $0.968+/-0.015$ & $0.963+/-0.025$ & $0.273+/-0.048$ & $0.853+/-0.070$ \\
\hline$X G B$ & $0.924+/-0.019$ & $0.957+/-0.011$ & $0.963+/-0.014$ & $0.952+/-0.029$ & $0.186+/-0.028$ & $0.820+/-0.083$ \\
\hline
\end{tabular}

AB, AdaBoost; GB, Gradient Booting; RF, Random Forests; XGB, eXtreme Gradient Boost; AUC, Area Under the Receiver Operating Characteristics Curve (ROC).

rate is $<1 \%$ during 5 -year follow-up after a normal MPI. In our application, the best model was RF, although GB and XGB had good results. RF was previously used successfully to predict mental problems in adolescents from specific questionnaires (AUC: 0.739) (37) and to forecast 1-, 2-, 3-, 4- and 5-year allcause mortality from pre-implant variables of patients submitted to cardiac resynchronization therapy (38), to foresee the outcome of 90Y-radioembolization in patients with intrahepatic tumors (39) and also to predict complete pathological response in rectal cancer after chemoradiotherapy using computed tomography radionics and 18F-fluorodeoxyglucose positron emission tomography (40) and Cantoni et al. (41) have evaluated the performance of SPECT and cadmium-zinc-telluride (CZT)SPECT in patients with CAD (or suspected) and have compared the diagnostic accuracy using RF. The sensitivity of CZT-SPECT and SPECT were 96 and 88\%, respectively. The main advantage of RF is its lower computational cost compared to Deep Learning, for example, generally eliminating Graphics Processing Units (GPUs). RF is a simple and powerful $\mathrm{ML}$ algorithm with applications even in other different contexts, such as the prediction of suicidal ideation (42) and right ventricular hypertrophy (43); however, its performance may vary depending, for example, on the application and the parameters used in the ML model. In assessing the first 5-year all-cause mortality separately, the results indicated an AUC that ranged between 0.76 and 0.8 , while in predicting the response to chemotherapy, the AUC obtained was $0.94(38,40)$. Also, no ML model is better than the others in all situations. Baskaran and colleagues, for instance, were successful in predicting obstructive coronary artery disease (AUC: 0.779) and revascularization (AUC: 0.958) from clinical and imaging data (44). Thus, it is usually interesting to analyze more than one model. Besides, the increase in the training database can contribute to making these algorithms' performance even better.

Besides the low computational effort for processing the algorithms, another advantage in our study was polar maps. A single two-dimensional image obtained during the stress, rest, or prone phase contains an adequate myocardium representation. However, some distortion may be induced in this process. In light of that, polar maps were used to obtain new images from the rotation (data augmentation) only in the training set, which resulted in a considerable expansion of the training databases in cross-validation. This increase in the database allows for an improved model training process and contributes to better results. It is an important alternative, especially when dealing with a database with an asymmetry of the outcomes-as highlighted by Thabtah and colleagues, a classification algorithm's performance can be affected if the study data is highly unbalanced (45). Kocheturov et al. emphasized that this asymmetry is considered a significant obstacle. It can lead to biased rules in favor of the majority of the result-which requires unique approaches to the issue (46). In our work, for example, images considered normal corresponded to about $10 \%$. However, after carrying out the data augmentation, this value increased to $32 \%$, which significantly improved the imbalance between classes.

Moreover, the storage size of images is small since each image is $<25 \mathrm{~KB}$. Another significant advantage is that our slicing process proved to be quite adequate to generate features. The use of vertical and horizontal slicing has already been used in different contexts. Shih et al., for instance, exhibited horizontal and vertical slices taking from 2 -day ${ }^{99 \mathrm{~m}} \mathrm{Tc}$-tetrofosmin SPECT images of a patient with duodenogastric reflux in a hiatal hernia (47). Teramoto et al. (48) used these slices to visualize the root system architecture of rice using X-ray computed tomography. Thus, this work brings as a novelty, not the issue of using vertical and horizontal cuts per se, but the use of this tool in generating attributes for ML models from polar maps. It was the first time that this type of slicing process was used in nuclear cardiology, to the best of our knowledge. The results obtained in this work suggest a potential use of this type of slicing. However, they do not exclude the possibility or validity of other types of approaches. Betancur et al. also took advantage of using polar maps. They developed successful neural networks (deep learning) 
to automatically predict obstructive coronary disease from MPI compared with current clinical methods (49). Togo et al. did a similar analysis using PET/CT images, also been successful with the use of polar maps. The idea was to assess the ML model (in case, deep learning) to distinguish between two different outcomes: cardiac sarcoidosis and non-cardiac sarcoidosis (50).

The excellent performance of our tool in the classification of polar maps has mainly two potential applications. The first one would be to analyze polar maps and compare them to the first report originated to assess any potential misinterpretation. Trägardh et al. (51) emphasized that reporting an image is usually the only form of communication between the physician and the caregiver, being one of the critical components of care delivery that can occasionally become legal evidence. Besides, suppose all images obtained during the stress, rest, and prone phase (in the case of a male patient) are considered normal in an ML algorithm evaluation. In that case, the final report should be normal, reducing the risk of mistakes in the medical report with the advantage of only analyzing 2 or 3 images. Also, the physician and ML model will act synergistically, as the model can identify potential errors. The specialist will identify false positives/negatives, which is essential for retraining and improving the models and their performance. Although the costs involved (financial, emotional, and others) could be significant, it seems that there are a few works related to this subject (reviewing previous evaluated reports). We also believe that ML tools could significantly differ in the medical training process in this context. For instance, medical residents can have an additional source to compare their reports, contributing to improving the learning process, therefore supporting their education.

\section{LIMITATIONS AND FUTURE WORK}

There are some limitations to this study: (a) it is essential to point out that we have collected all data retrospectively from a single center. Although the benefits of using ten-fold cross-validation, deriving a predictive model from historical data may affect the generalization of the results since we could have seen changes in the patient's characteristics as time goes by $(1,52)$. (b) A few experts were responsible for producing the medical report. (c) We used a 2-days protocol. So, we may not be able to use this model for a 1-day protocol. In light of that, it is essential to validate our results in different data and distinct contexts. (d) We did not use any clinical information, which could improve the models' performance. In future work, we believe that the methodology developed here can be applied to other contexts, including polar maps obtained with different radiotracers or even

\section{REFERENCES}

1. Alonso DH, Wernick MN, Yang Y, Germano G, Berman DS, Slomka P. Prediction of cardiac death after adenosine myocardial perfusion SPECT based on machine learning. J Nucl Cardiol. (2019) 26:1746-54. doi: 10.1007/s12350-018-1250-7 different outcomes, such as the definition of the territory where the abnormality was verified. (e) Possibly the originality of the proposed method could be explained because of our dimensional reduction method. In this context, different types of reduction methods, such as principal component analysis (PCA) (53) and independent component analysis (ICA) (54) could improve our results and should be explored in future work. (f) The model has a focus on predicting only whether the MPI is normal or abnormal.

We did not provide any information on the ischemic heart area and the patient's prognosis. Despite this, we believe that our tool could also help optimize and prioritize reporting queues.

\section{CONCLUSIONS}

We have successfully implemented 4 ensemble ML algorithms (RF, GB, XGB, AB) to distinguish normal vs. abnormal SPECT myocardial perfusion polar maps. We used 10 different features extracted using an image slicing process and ten-fold cross-validation. Data augmentation was done in the training set, considering the polar maps' geometric properties and rotating normal images through small angles. The computational times were very low, and RF had the best AUC. We believe that our tool can contribute to a reevaluation of previously reported images and a medical training process for residents, identifying possible mistakes.

\section{DATA AVAILABILITY STATEMENT}

The original contributions presented in the study are included in the article/Supplementary Material, further inquiries can be directed to the corresponding authors.

\section{AUTHOR CONTRIBUTIONS}

ES, FF, CM, and RG: conception or design of the work. ES, FF, TS, and CM: data collection. ES, FS, and LC: data analysis. ES, FS, CM, RG, CW, and BC: interpretation. ES, FF, CW, BC, FS, $\mathrm{CM}$, and RG: drafting the article. AS and EM: critical revision of the article. ES, FS, CM, and RG: final approval of the version to be published. All authors contributed to the article and approved the submitted version.

\section{SUPPLEMENTARY MATERIAL}

The Supplementary Material for this article can be found online at: https://www.frontiersin.org/articles/10.3389/fcvm. 2021.741667/full\#supplementary-material 
4. Tilkemeier PL, Bourque J, Doukky R, Sanghani R, Weinberg RL. ASNC imaging guidelines for nuclear cardiology procedures. J Nucl Cardiol. (2017) 24:2064-128. doi: 10.1007/s12350-017-1057-y

5. Henzlova MJ, Duvall WL. Temporal changes in cardiac SPECT utilization and imaging findings: where are we going and where have we been? J Nucl Cardiol. (2020) 27:2178-82. doi: 10.1007/s12350-019-01687-y

6. Porter ME. A strategy for health care reform - toward a value-based system. N Engl J Med. (2009) 361:109-12. doi: 10.1056/NEJMp0904131

7. Souza Filho EM, Fernandes FA, Soares CLA, Seixas FL, Santos AASMDD, Gismondi RA, et al. Artificial intelligence in cardiology: concepts, tools and challenges - "the horse is the one who runs, you must be the jockey". Arq Bras Cardiol. (2020) 114:718-25. doi: 10.36660/abc.2018043

8. Iannattone PA, Zhao X, VanHoutten J, Garg A, Huynh T. Artificial intelligence for diagnosis of acute coronary syndromes: a meta-analysis of mixed methods of machine learning. Can J Cardiol. (2019) 36:577-83. doi: 10.1016/j.cjca.2019.09.013

9. Massalha S, Clarkin O, Thornhill R, Wells G, Chow BJW. Decision support tools, systems, and artificial intelligence in cardiac imaging. Can J Cardiol. (2018) 34:827-38. doi: 10.1016/j.cjca.2018.04.032

10. Henzlova MJ, Duvall WL, Einstein AJ, Travin MI, Verberne HJ. ASNC imaging guidelines for SPECT nuclear cardiology procedures: stress, protocols, and tracers. J Nucl Cardiol. (2016) 23:606-39. doi: 10.1007/s12350-015-0387-x

11. Hudson HM, Larkin RS. Accelerated image reconstruction using ordered subsets of projection data. IEEE Trans Med Imaging. (1994) 13:601-9. doi: $10.1109 / 42.363108$

12. Dorbala S, Ananthasubramaniam K, Armstrong IS, Chareonthaitawee P, DePuey EG, Einstein AJ, et al. Single photon emission computed tomography (SPECT) myocardial perfusion imaging guidelines: instrumentation, acquisition, processing, and interpretation. J Nucl Cardiol. (2018) 25:1784-846. doi: 10.1007/s12350-018-1283-y

13. Ouali C, Dumouchel P, Gupta V. A spectrogram-based audio fingerprinting system for content-based copy detection. Multimed Tools Appl. (2016) 75:9145-65. doi: 10.1007/s11042-015-3081-8

14. GDPR. Regulation (eu) 2016/679 of the European Parliament and of the Council of 27 April 2016:on the protection of natural persons with regard to the processing of personal data and on the free movement of such data, and repealing Directive 95/46/EC (General Data Protection Regulation). Official Journal of the European Union, L 119, (2016).

15. Wang CW. New ensemble machine learning method for classification and prediction on gene expression data. Conf Proc IEEE Eng Med Biol Soc. New York, NY, (2006) 2006:3478-81. doi: 10.1109/IEMBS.2006.259893

16. Freund Y, Schapire RE. A decision-theoretic generalization of on-line learning and an application to boosting. J Comput Syst Sci. (1997) 55:119-39. doi: $10.1006 /$ jcss.1997.1504

17. Hastie T, Tibshirani R, Friedman J. The Elements of Statistical Learning: Data Mining, Inference, and Prediction. 2nd ed. New York, NY: Springer (2009). ISBN 978-0-387-84858-7. doi: 10.1007/978-0-387-84858-7

18. Agarwal P, Tang J, Narayanan ANL, Zhuang J. Big data and predictive analytics in fire risk using weather data. Risk Anal. (2020) 40:1438-49. doi: $10.1111 /$ risa. 13480

19. Breiman L. Arcing the edge. Technical Report 486. Statistics Department. University of California, Berkeley. (1997).

20. Friedman JH. Greedy function approximation: a gradient boosting machine. Ann Stat. (2001) 29:1189-232. doi: 10.1214/aos/1013203451

21. Friedman JH. Stochastic gradient boosting. Comput Stat Data Anal. (2002) 38:367-78. doi: 10.1016/S0167-9473(01)00065-2

22. Chen T, Guestrin C. XGBoost: A Scalable Tree Boosting System. In: Krishnapuram B, Shah M, Smola AJ., editors. Proceedings of the 22nd ACM SIGKDD International Conference on Knowledge Discovery and Data Mining. San Francisco, CA, USA (2016). p. 785-94. doi: 10.1145/2939672.29 39785

23. Breiman L. Random forests. Mach Learn. (2001) 45:5-32. doi: 10.1023/A:1010933404324

24. Ho TK. Random decision forests. In: Proceedings of the 3rd International Conference on Document Analysis and Recognition. Montreal. Washington, DC: IEEE Computer Society (1995). p. 278-82.

25. Python Software Foundation. Python Language Reference version 3. Available online at http://www.python.org/
26. Pedregosa F, Varoquaux G, Gramfort A, Michel V. Thirion B. Scikit-learn: machine learning in python. J Mach Learn Res. (2011) 12:2825-30. Available online at: https://jmlr.org/papers/volume12/pedregosa11a/pedregosa11a.pdf

27. Kakadiaris IA, Vrigkas M, Yen AA, Kuznetsova T, Budoff M, Naghavi M. Machine learning outperforms ACC / AHA CVD risk calculator in MESA. J Am Heart Assoc. (2018) 7:e009476. doi: 10.1161/JAHA.118.009476

28. Shiri I, Sabet KA, Arabi H, Pourkeshavarz M, Teimourian B, Ay MR, et al. Standard SPECT myocardial perfusion estimation from half-time acquisitions using deep convolutional residual neural networks. J Nucl Cardiol. (2020). doi: 10.1007/s12350-020-02119-y. [Epub ahead of print].

29. Nakajima K, Kudo T, Nakata T, Kiso K, Kasai T, Taniguchi Y, et al. Diagnostic accuracy of an artificial neural network compared with statistical quantitation of myocardial perfusion images: a Japanese multicenter study. Eur J Nucl Med Mol Imaging. (2017) 44:2280-9. doi: 10.1007/s00259-017-3834-x

30. Rosenblatt F. The perceptron: a probabilistic model for information storage and organization in the brain. Psychol Rev. (1958) 65:386-408. doi: 10.1037/h0042519

31. LeCun Y, Bengio Y, Hinton G. Deep learning. Nature. (2015) 521:436. doi: 10.1038/nature14539

32. Rumelhart DE, Hinton GE, Williams RJ. Learning representations by backpropagating errors. Nature (1986) 323:533-6. doi: 10.1038/323533a0

33. Arsanjani R, Dey D, Khachatryan T, Shalev A, Hayes SW, Fish M, et al. Prediction of revascularization after myocardial perfusion SPECT by machine learning in a large population. J Nucl Cardiol. (2015) 22:877-84. doi: $10.1007 /$ s12350-014-0027-x

34. Friedman J, Hastie T, Tibshirani R. Additive logistic regression: a statistical view of boosting. Ann Stat. (2000) 28:337-407. doi: 10.1214/aos/1016218223

35. Cortes C, Vapnik V. Support-vector networks. Mach Learn. (1995) 20:273-97. doi: 10.1007/BF00994018

36. Elhendy A, Schinkel A, Bax JJ, van Domburg RT, Poldermans D. Long-term prognosis after a normal exercise stress Tc-99m sestamibi SPECT study. J Nucl Cardiol. (2003) 10:261-6. doi: 10.1016/S1071-3581(02)43219-9

37. Tate AE, McCabe RC, Larsson H, Lundström S, Lichtenstein P, Kuja-Halkola R. Predicting mental health problems in adolescence using machine learning techniques. PLoS ONE. (2020) 15:e0230389. doi: 10.1371/journal.pone.0230389

38. Tokodi M, Schwertner WR, Kovács A, Tosér Z, Staub L, Sárkány A, et al. Machine learning based mortality prediction of patients undergoing cardiac resynchronization therapy: the SEMMELWEIS-CRT score. Eur Heart J. (2020) 41:1747-56. doi: 10.1093/eurheartj/ehz902

39. Ingrisch M, Schöppe F, Paprottka K, Fabritius M, Strobl FF, De Toni EN, et al. Prediction of $90 \mathrm{Y}$ radioembolization outcome from Pretherapeutic factors with random survival forests. J Nucl Med. (2018) 59:769-73. doi: 10.2967/jnumed.117.200758

40. Shen WC, Chen SW, Wu KC, Lee PY, Feng CL, Hsieh TC, et al. Predicting pathological complete response in rectal cancer after chemoradiotherapy with a random forest using 18F-fluorodeoxyglucose positron emission tomography and computed tomography radiomics. Ann Transl Med. (2020) 8:207. doi: 10.21037/atm.2020.01.107

41. Cantoni V, Green R, Ricciardi C, Assante R, Zampella E, Nappi C, et al. A machine learning-based approach to directly compare the diagnostic accuracy of myocardial perfusion imaging by conventional and cadmium-zinc telluride SPECT. J Nucl Cardiol. (2020). doi: 10.1007/s12350-020-02187-0. [Epub ahead of print].

42. Lin GM, Nagamine M, Yang SN, Tai YM, Lin C, Sato H. Machine learning based suicide ideation prediction for military personnel. IEEE J Biomed Health Inform. (2020) 24:1907-16. doi: 10.1109/JBHI.2020.2988393

43. Lin GM, Lu HH. A 12-lead ecg-based system with physiological parameters and machine learning to identify right ventricular hypertrophy in young adults. IEEE J TranslEng Health Med. (2020) 8:1900510. doi: 10.1109/JTEHM.2020.2996370

44. Baskaran L, Ying X, Xu Z, Al'Aref SJ, Lee BC, Lee SE, et al. Machine learning insight into the role of imaging and clinical variables for the prediction of obstructive coronary artery disease and revascularization: an exploratory analysis of the CONSERVE study. PLoS ONE. (2020) 15:e0233791. doi: 10.1371/journal.pone.0233791

45. Thabtah F, Hammoud S, Kamalov F, Gonsalves A. Data imbalance in classification: experimental evaluation. Inf Sci. (2020) 513:429-41. doi: 10.1016/j.ins.2019.11.004 
46. Kocheturov A, Pardalos PM, Karakitsiou A. Massive datasets and machine learning for computational biomedicine: trends and challenges. Ann Oper Res. (2019) 276:5-34. doi: 10.1007/s10479-0182891-2

47. Shih WJ, Shih GL, Huang WS, Milan P. Duodenogastric reflux in a hiatal hernia seen as retrocardiac activity on 99mTc-tetrofosmin cardiac SPECT raw-data images. J Nucl Med Technol. (2007) 35:252-4. doi: $10.2967 /$ jnmt.107.041178

48. Teramoto S, Takayasu S, Kitomi Y, Arai-Sanoh Y, Tanabata T, Uga Y. High-throughput three-dimensional visualization of root system architecture of rice using X-ray computed tomography. Plant Methods. (2020) 16:66. doi: 10.1186/s13007-020-00612-6

49. Betancur J, Commandeur F, Motlagh M, Sharir T, Einstein AJ, Bokhari S, et al. Deep learning for prediction of obstructive disease from fast myocardial perfusion SPECT: a multicenter study. JACC Cardiovasc Imaging. (2018) 11:1654-63. doi: 10.1016/j.jcmg.2018. 01.020

50. Togo R, Hirata K, Manabe O, Ohira H, Tsujino I, Magota K, et al. Cardiac sarcoidosis classification with deep convolutional neural networkbased features using polar maps. Comput Biol Med. (2019) 104:81-6. doi: 10.1016/j.compbiomed.2018.11.008

51. Trägårdh E, Hesse B, Knuuti J, Flotats A, Kaufmann PA, Kitsiou A, et al. Reporting nuclear cardiology: a joint position paper by the European association of nuclear medicine (EANM) and the european association of cardiovascular imaging (EACVI). Eur Heart J Cardiovasc Imaging. (2015) 16:272-9. doi: 10.1093/ehjci/jeu304

52. Rozanski A, Gransar H, Hayes SW, Min J, Friedman JD, Thomson LE, et al. Temporal trends in the frequency of inducible myocardial ischemia during cardiac stress testing: 1991 to 2009. J Am Coll Cardiol. (2013) 61:1054-65. doi: 10.1016/j.jacc.2012.11.056

53. Miyoshi T, Higaki A, Kawakami H, Yamaguchi O. Automated interpretation of the coronary angioscopy with deep convolutional neural networks. Open Heart. (2020) 7:e001177. doi: 10.1136/openhrt-2019-001177

54. Stone, James V. Independent Component Analysis: A Tutorial Introduction. Cambridge, Massachusetts: MIT Press. ISBN 978-0-262-69315-8. (2004). doi: $10.7551 /$ mitpress/3717.001.0001

Conflict of Interest: The authors declare that the research was conducted in the absence of any commercial or financial relationships that could be construed as a potential conflict of interest.

Publisher's Note: All claims expressed in this article are solely those of the authors and do not necessarily represent those of their affiliated organizations, or those of the publisher, the editors and the reviewers. Any product that may be evaluated in this article, or claim that may be made by its manufacturer, is not guaranteed or endorsed by the publisher.

Copyright (c) 2021 de Souza Filho, Fernandes, Wiefels, de Carvalho, dos Santos, dos Santos, Mesquita, Seixas, Chow, Mesquita and Gismondi. This is an open-access article distributed under the terms of the Creative Commons Attribution License (CC $B Y)$. The use, distribution or reproduction in other forums is permitted, provided the original author(s) and the copyright owner(s) are credited and that the original publication in this journal is cited, in accordance with accepted academic practice. No use, distribution or reproduction is permitted which does not comply with these terms. 